\title{
$O$ efeito da eritropoetina humana recombinante no tratamento da anemia da prematuridade
}

\author{
The effect of recombinant human erythropoietin on the treatment of anemia of prematurity
}

\author{
Vera Lúcia L. Rocha ${ }^{1}$, Ana Cláudia W. Benjamin ${ }^{2}$, Renato S. Procianoy ${ }^{3}$
}

\section{Resumo}

Objetivos: a proposta do presente estudo foi avaliar a eficácia da eritropoetina na prevenção e tratamento da anemia da prematuridade, correlacionar o uso desta medicação com o ganho de peso, comprimento e perímetro cefálico dos pacientes. Foi realizada também uma comparação entre o uso de eritropoetina diariamente e o uso duas vezes por semana, na mesma dose semanal.

Métodos: ensaio clínico que avaliou 42 recém-nascidos prematuros, com até 33 semanas de idade gestacional, peso de nascimento até $1550 \mathrm{~g}$ e idade pós-natal entre 10 e 35 dias de vida. Os recém-nascidos foram randomizados em três grupos. Os pacientes do grupo 1 receberam 7 doses diárias, de 100U/kg cada, de eritropoetina, por semana, enquanto nos pacientes do grupo 2 foram administradas duas doses, na semana, de $350 \mathrm{U} / \mathrm{kg}$ cada, e os pacientes do grupo 3 não receberam a medicação. Durante o tratamento foram quantificados índices hematológicos, as transfusões sangüíneas realizadas e o crescimento das crianças.

Resultados: os bebês dos grupos tratados e os do grupo controle não apresentaram diferença quanto ao peso, ao comprimento, ao perímetro cefálico e ao tempo de internação. Ao término do estudo não foi observada nenhuma diferença estatisticamente significativa quanto às médias dos valores das plaquetas, leucócitos totais e ferritina entre os três grupos estudados. Já a média final do hematócrito e da hemoglobina dos pacientes que não receberam a eritropoetina foi significativamente menor em relação àqueles que receberam a medicação. A média da contagem absoluta de reticulócitos, no final da segunda semana de tratamento, foi significativamente maior nos pacientes que receberam a medicação, quando comparada à dos que não utilizaram a eritropoetina. Os grupos 1 e 2 foram significativamente menos transfundidos excessivamente (2 ou mais ) que o grupo 3. A administração de eritropoetina, na dose de 700/kg/semana diminuiu de forma significativa o número de transfusões excessivas. Não houve diferença significativa quanto à quantidade de sangue transfundido entre os pacientes que recebem a eritropoetina diariamente quando comparados aos que recebem a droga duas vezes por semana.

Conclusões: o uso da eritropoetina não influenciou o ganho de peso e o crescimento. A eritropoetina administrada na dose semanal de 700UI/ $\mathrm{kg}$ em prematuros com peso até $1550 \mathrm{~g}$ e idade gestacional de até 33 semanas estimula a eritropoese e reduz de forma significativa o número de transfusões sanguíneas excessivas. Esta medicação mostrou ser segura, bem tolerada e sem paraefeitos a curto prazo.

J Pediatr (Rio J) 2001; 77 (2): 75-83: eritropoetina, anemia, prematuro.

\begin{abstract}
Objective: to assess the efficacy of erythropoietin in the prevention and treatment of anemia of prematurity, correlating the use of this drug with weight gain, length, and head circumference and comparing two administration schemes of he same weekly dose: daily use and twice a week.
\end{abstract}

Methods: the study comprised 42 premature newborns with gestational age up to 33 weeks, birthweight up to $1550 \mathrm{~g}$, and postnatal age between 10 and 35 days. The newborns were randomized into three groups: patients in group 1 received seven daily doses of $100 \mathrm{U} / \mathrm{kg}$ erythropoietin per week; patients in group 2 received two $350 \mathrm{U} / \mathrm{kg}$ erythropoietin doses per week; and patients in group 3 did not receive the drug. Hematologic measurements, blood transfusion requirements, and growth rates were followed during therapy.

Results: cases and controls did not differ with respect to weight, length, head circumference, and total time of hospital stay. At the end of the study, no significant difference was observed in the platelet count measurement means, white blood cell count, and ferritin levels in the three groups. However, the final hematocrit and hemoglobin values of patients who did not receive erythropoietin were significantly lower than those of patients who received the drug. The absolute reticulocyte count mean was significantly higher in patients who received erythropoietin after two weeks of treatment when compared with those patients who did not receive the drug. Patients in group 1 e 2 received fewer excessive transfusions ( 2 or more) than patients in group 3. The administration of $700 \mathrm{U} / \mathrm{kg} /$ week erythropoietin significantly reduced the number of excessive blood transfusions. There is no significant difference in blood transfusion volume between patients who received erythropoietin on a daily basis and those who received the drug twice weekly.

Conclusions: the use of erythropoietin did not influence weight gain and growth. The administration of $700 \mathrm{U} / \mathrm{kg} /$ week erythropoietin in premature infants with gestational age up to 33 weeks and birthweight up to $1550 \mathrm{~g}$ stimulates erythropoiesis and significantly reduces excessive blood transfusion requirements. Erythropoietin showed to be a safe and well tolerated medication, with no short-term side effects in the study population.

J Pediatr (Rio J) 2001; 77 (2): 75-83: erythropoietin, anemia of prematurity, transfusion, premature.

1. Médica neonatologista do Hosp. de Clínicas de Porto Alegre (HCPA) e do Hospital-Escola Materno Infantil Pres. Vargas, Mestre em Pediatria pela UFRGS.

2. Médica neonatologista, Mestranda em Pediatria pela UFRGS.

3. Professor titular de Pediatria da Faculdade de Medicina da UFGRS. Chefe da Unidade de Neonatologia do HCPA. Coordenador do curso de pós-graduação em Pediatria da Faculdade de Medicina da UFRGS. 


\section{Introdução}

Durante as primeiras semanas de vida, todos os RNs apresentam declínio dos glóbulos vermelhos, o que representa uma adaptação fisiológica ao ambiente extra-uterino ${ }^{1-3}$. Nos prematuros essa diminuição da hemoglobina $(\mathrm{Hb})$ é mais rápida ${ }^{4,5}$, e seu valor mínimo é mais baixo ${ }^{6}$ quando comparado ao dos RNs a termo ${ }^{2}$, denominando-se de anemia da prematuridade. Ela é um processo autolimitado, e sua intensidade aumenta quanto mais prematuro for $\mathrm{o}$ $\mathrm{RN}$, tornando-se, assim, menos fisiológica ${ }^{7}$. Os mecanismos pelos quais a anemia da prematuridade se desenvolve são coleta excessiva de sangue para exames laboratoriais, crescimento somático das crianças, menor vida média dos eritrócitos neonatais, pequena transfusão placento-fetal ao nascimento e cessação da liberação de eritropoetina $(\mathrm{EPO})^{6,8-11}$. Vários estudos encontraram, neste período, concentrações séricas de EPO inapropriadamente bai$\mathrm{xas}^{5,7,12}$. No prematuro, essa supressão persiste por semanas e, apesar da gravidade da anemia, na maioria destes pacientes a EPO não é liberada antes da $30^{\mathrm{a}}$ a $34^{\mathrm{a}}$ semanas ${ }^{13}$.

No tratamento da anemia da prematuridade a transfusão sangüínea (TS) é o método mais comumente utilizado ${ }^{14}$. Estima-se que dos, aproximadamente, $38.000 \mathrm{RNs}$ por ano, nos Estados Unidos, com peso de nascimento até $1500 \mathrm{~g}$, $80 \%$ receberão múltiplas transfusões sangüíneas ${ }^{15}$. Apesar de todos os cuidados com as TSs, esse não é um procedimento totalmente seguro, havendo várias complicações possíveis, como hemólise, hipercalemia, descompensação cardiopulmonar por sobrecarga de volume em pacientes muito pequenos e infecções $7,8,16-19$. Além dessas potenciais complicações, as TSs precoces de RNs criticamente doentes fazem com que a $\mathrm{Hb}$ fetal seja substituída pela do tipo adulto, que tem uma menor afinidade com o oxigênio, transportando-o mais prontamente aos tecidos e diminuindo, assim, o estímulo para a produção de $\mathrm{EPO}^{8,20}$.

O sucesso do uso de EPO no tratamento da anemia de adultos e crianças com insuficiência renal crônica desencadeou vários ensaios clínicos em que foi testado o uso da mesma na anemia da prematuridade ${ }^{8}$. Shannon e colaboradores $^{21}$, em 1987, e Rhondeau e colaboradores ${ }^{20}$, em 1988, confirmaram a presença de progenitores eritróides no sangue periférico de RNs com anemia da prematuridade e com respostas normais intrínsecas à EPO. Em 1990, um relato de 7 casos mostrou que a EPO associada a doses adequadas de ferro pode acelerar a recuperação da anemia da prematuridade $^{22}$. No ano seguinte dois ensaios clínicos randomizados não mostraram melhora na $\mathrm{Hb}$ dos pacientes tratados com $\mathrm{EPO}^{3,23}$. Em 1993 e 1995, Soubasi e colaboradores mostraram que há sucesso com o uso de EPO na anemia da prematuridade ${ }^{10,23}$. Outros estudos demonstraram menor necessidade de TSs associadas ao tratamento com $\mathrm{EPO}^{24,25}$. Bem recentemente, em 1999, Brown e colaboradores concluíram que a EPO, quando administrada mais freqüentemente, produz um aumento no estímulo da eritropoese do $\mathrm{RN}$ de muito baixo peso ${ }^{26}$.
Tendo em vista a importância da anemia da prematuridade e de seu tratamento, foi realizado este ensaio clínico, com o propósito de avaliar a eficácia da EPO no seu tratamento, comparar dois esquemas terapêuticos - o uso diário de EPO e o uso duas vezes por semana, ambos com a mesma dose semanal, e também verificar a existência de associação entre o desenvolvimento pondero-estatural e o uso da EPO.

\section{Material e Métodos}

Este ensaio clínico foi realizado na Unidade de Neonatologia do Hospital de Clínicas de Porto Alegre (HCPA). A análise do sangue coletado durante o estudo foi realizada nos laboratórios de hematologia e radioimunoensaio do próprio HCPA. O projeto foi aprovado pelas Comissões Científica e Ética em Saúde, do grupo de pesquisa e pósgraduação do HCPA, tendo sido considerado metodológica e eticamente adequado de acordo com as Diretrizes e Normas Regulamentadoras de Pesquisa Envolvendo Seres Humanos. Após o consentimento do médico assistente e o consentimento por escrito dos pais ou responsáveis pelo paciente, os PMTs que preencheram os seguintes critérios de inclusão foram admitidos no estudo: idade gestacional de até 33 semanas; peso de nascimento de até $1550 \mathrm{~g}$; idade pós-natal entre 10 e 35 dias de vida; contagem de plaquetas superior a $50.000 \mathrm{~mm}^{3} ; \mathrm{Ht}$ inicial acima de $32 \%$; ausência de convulsões ou hemorragia cerebral acima de grau II; ausência de história de anemia hemolítica causada por incompatibilidade $\mathrm{ABO}, \mathrm{RH}$ ou outras e estabilidade clínica caracterizada por alimentação enteral de pelo menos dois terços das necessidades calóricas diárias, ausência de infecções congênitas ou adquiridas e necessidade de suporte ventilatório mínimo (definido por até $30 \%$ de oxigênio, $20 \mathrm{mvm}$ de frequiência respiratória, $20 \mathrm{~cm} \mathrm{de}_{2} \mathrm{O}$ de pressão inspiratória e $4 \mathrm{~cm}$ de $\mathrm{H}_{2} \mathrm{O}$ de pressão expiratória). Nesta ocasião cada paciente foi alocado de forma sistemática a um dos três grupos existentes: grupo 1, formado pelos pacientes que receberam a EPO diariamente; grupo 2, composto pelos pacientes que receberam a EPO duas vezes por semana; grupo 3 (controles), integrado pelos pacientes que não receberam a medicação testada.

A seguir foi preenchido, para cada paciente, um protocolo com dados de identificação, data e peso de nascimento e idade gestacional. Foram coletados exames iniciais: hemograma, plaquetas, reticulócitos e ferritina. Os três primeiros foram repetidos semanalmente, sendo a ferritina dosada uma vez a cada quinze dias. Todo o volume de sangue retirado para os testes laboratoriais e todo o volume e o número de TSs realizadas em cada paciente foram registrados. A indicação de transfusão foi realizada, em toda a população estudada, pelo médico assistente de cada $\mathrm{RN}$, baseada nos seguintes critérios: $\mathrm{Ht} \leq 20 \%$, ganho de peso inadequado, três ou mais episódios de apnéia ou bradicardia em 24 horas, necessidade pré-procedimento cirúrgico, doença associada à queda súbita do Ht, reposição 
de sangue retirado para exames laboratoriais, manutenção do $\mathrm{Ht}$ de até $30 \%$ associado à necessidade de suporte ventilatório mínimo e Ht de até 35\% quando a necessidade ventilatória é maior. O médico assistente que indicou as TSs desconhecia a que grupo do estudo o paciente pertencia. Os PMTs que participaram do estudo foram avaliados diariamente, através de exame físico, controle dos sinais vitais, balanço hídrico e peso. Semanalmente foram medidos o perímetro cefálico e o comprimento.

O medicamento utilizado durante o estudo foi a eritropoetina humana recombinante em concentração de 2000U/ $\mathrm{ml}$. Os pacientes do grupo 1 receberam o medicamento diariamente, na dose de $100 \mathrm{U} / \mathrm{kg} / \mathrm{dia}$; os do grupo 2 , duas vezes por semana, na dose de $350 \mathrm{U} / \mathrm{kg} / \mathrm{dia}$ e os do grupo 3 não receberam a medicação. A medicação foi administrada até o RN completar $2 \mathrm{~kg}$. Os grupos $1 \mathrm{e} 2$, portanto, utilizaram a mesma dose semanal, mas em diferentes esquemas terapêuticos. A via de administração utilizada foi a subcutânea. O sulfato ferroso foi utilizado nos grupos 1 e 2, via oral, por sonda orogástrica ou nasogástrica, na dose de $3 \mathrm{mg} / \mathrm{kg} / \mathrm{dia}$, desde a primeira semana do estudo, sendo aumentada para $6 \mathrm{mg} / \mathrm{kg} / \mathrm{dia}$ a partir da segunda semana de tratamento. No grupo 3, essa suplementação de ferro iniciou de acordo com a rotina do médico assistente do paciente, em torno de 30 dias de vida. Todos os PMTs do estudo receberam vitaminas $\mathrm{A}, \mathrm{C}, \mathrm{D}$ e $\mathrm{E}$, nas doses de 2000UI, 35mg, 400UI e 20U por dia, respectivamente.

A coleta de dados foi interrompida após ter sido realizado um cálculo de amostra evidenciando que para obtenção de uma diferença estastística entre os dois grupos de PMTs que usaram a EPO em esquemas posológicos diferentes seria necessário, no mínimo, o dobro do número de pacientes estudados até aquele momento. Os autores se satisfizeram, portanto, quando foi encontrada diferença estatisticamente significativa entre os dois grupos tratados e o grupo controle quanto ao número de TSs excessivas. Inicialmente foi realizada uma avaliação das características da população estudada através de uma análise descritiva. Para análise das variáveis contínuas foram calculadas a média, a mediana, o desvio padrão e a amplitude interquartil, de acordo com a presença ou não de assimetria nos dados. Para variáveis categóricas foram calculados percentuais. $\mathrm{Na}$ comparação das variáveis contínuas entre os grupos foi utilizado o teste ANOVA, com localização de diferenças significativas pelo teste de Tukey. As variáveis com assimetria foram comparadas pelo teste de Kruskal-Wallis. As variáveis categóricas e algumas contínuas foram categorizadas e comparadas em tabelas de contingência através do teste Qui-quadrado. Adicionalmente foram calculados coeficientes de correlação de Pearson para avaliar a associação entre as variáveis categóricas. $O$ tratamento estatístico dos dados foi realizado com a utilização do programa computadorizado SPSS (Statistical Package for Social Science). Em todos os testes, foi estabelecido o nível de significância de alfa $=0,05$.

\section{Resultados}

Foram selecionados, no período de março de 1995 a dezembro de 1996, a partir do preenchimento de todos os critérios de inclusão do estudo, 45 recém-nascidos, todos internados na Unidade de Neonatologia do HCPA. Três deles (um do grupo 2 e dois do grupo 3 ) não foram incluídos na análise dos dados por terem apresentado intercorrências clínicas ( 2 casos de sepse grave e 1 de enterocolite necrotizante), o que impediu sua participação no estudo. Foram portanto analisados 42 PMTs: 15 do grupo 1, 14 do grupo 2 e 13 do grupo 3.

As características gerais estudadas podem ser vistas na Tabela 1. Todas as características distribuíram-se de forma semelhante entre os grupos, sem diferenças estatisticamente significativas. Não houve diferença significativa entre o número de RNs pequenos e adequados para a idade gestacional.

$\mathrm{Na}$ avaliação clínica dos pacientes, observou-se uma estabilidade em todos os grupos. A administração da EPO, via subcutânea, foi bem tolerada por todos os PMTs do estudo. Não foram observados efeitos colaterais nos grupos (1 e 2) que receberam a medicação testada.

Tabela 1 - Características gerais da população com relação a peso, comprimento e perímetro cefálico ao nascimento, idade gestacional e idade pós-natal na entrada do estudo

\begin{tabular}{|c|c|c|c|c|c|c|c|}
\hline \multirow[t]{2}{*}{ Características } & \multicolumn{2}{|c|}{$\begin{array}{c}\text { Grupo 1 } \\
\mathrm{n}=15\end{array}$} & \multicolumn{2}{|c|}{$\begin{array}{c}\text { Grupo } 2 \\
\mathrm{n}=14\end{array}$} & \multicolumn{2}{|c|}{$\begin{array}{c}\text { Grupo } 3 \\
\mathbf{n}=\mathbf{1 3}\end{array}$} & \multirow[t]{2}{*}{$\mathbf{P}^{*}$} \\
\hline & Média & DP & Média & DP & Média & DP & \\
\hline Peso ao nascimento (g) & 1191 & 261,45 & 1173,93 & 272,24 & 1182,31 & 245,87 & 0,985 \\
\hline Idade gestacional (semanas) & 31,33 & 1,88 & 31,71 & 1,60 & 31 & 1,13 & 0,810 \\
\hline Peso inicial (g) & 1181,3 & 219,3 & 1164,3 & 268,8 & 1176,9 & 68,9 & 0,981 \\
\hline Comprimento inicial (cm) & 37,9 & 2,86 & 37,44 & 2,78 & 37,92 & 2,91 & 0,882 \\
\hline Perímetro cefálico inicial (cm) & 26,93 & 1,66 & 27,36 & 2,05 & 27 & 1,86 & 0,811 \\
\hline Idade inicial (dias) & 16,13 & 6,97 & 14,42 & 5,54 & 15,4 & 5,3 & 0,749 \\
\hline
\end{tabular}


Não houve diferença estatisticamente significativa entre os grupos quanto ao ganho de peso, comprimento ou perímetro cefálico dos pacientes. A média de peso dos pacientes no término do trabalho também foi semelhante entre os grupos 1,2 e 3. A Tabela 2 mostra a média de peso, do comprimento e do perímetro cefálico dos três grupos, na entrada e no final do estudo, bem como o aumento médio dessas três variáveis descritas.

Os três grupos estudados foram semelhantes quanto aos aportes calórico e protéico administrados, utilizando em média, respectivamente, $122 \mathrm{cal} / \mathrm{kg} / \mathrm{dia}$ e $2,7 \mathrm{~g} / \mathrm{kg} / \mathrm{dia}$.

$\mathrm{Na}$ Tabela 3 estão as médias dos valores de $\mathrm{Ht}, \mathrm{Hb}$, reticulócitos, leucócitos totais, plaquetas e ferritina sérica dos três grupos no início do estudo. Todas essas variáveis foram estatisticamente semelhantes no momento referido do trabalho.

Na Tabela 4 se encontram as médias dos valores de Ht, $\mathrm{Hb}$, ferritina e reticulócitos, dos três grupos, no término do estudo e dos reticulócitos no término da segunda semana de tratamento.

O resultado da avaliação da média do Ht final evidenciou uma diferença significativa entre os pacientes que receberam a EPO e os que não fizeram uso da medicação. Nos pacientes dos grupos 1 e 2 que receberam a medicação diariamente ou duas vezes por semana, respectivamente, a média final do hematócrito foi significativamente maior, quando comparada à do grupo 3 (controle).

Quanto à média da $\mathrm{Hb}$ ao término do seguimento dos pacientes, o menor valor foi detectado no grupo 3: 8,3. A análise estatística mostrou diferença significativa entre os grupos. Observa-se uma diminuição significativamente maior da $\mathrm{Hb}$ nos pacientes que não receberam a medicação testada (grupo 3) em relação aos grupos 1 e 2.

Em relação à contagem de leucócitos totais, não houve diferença significativa entre os grupos, em nenhuma das semanas estudadas. $\mathrm{Na}$ avaliação dos neutrófilos não foi constatada diferença entre os grupos tratados ( 1 e 2) e o que não recebeu a medicação (grupo 3) em nenhuma das sema- nas estudadas. No tocante à contagem de plaquetas, também não foi observada diferença significativa entre os grupos em nenhuma das semanas estudadas.

Foram realizados testes estatísticos para analisar a variável ferritina. Na primeira testagem não foi detectada nenhuma diferença significativa entre os grupos em nenhuma das semanas estudadas. Como se trata de uma variável assimétrica, testes não paramétricos foram realizados a fim de comprovar o resultado encontrado. Ainda assim, o resultado dos primeiros testes - ausência de diferença significativa - permaneceu, sendo o valor do $\mathrm{p}$ dos testes não paramétricos, de 0,344 .

A média da contagem absoluta de reticulócitos, no final da segunda semana de tratamento, foi significativamente maior nos pacientes que receberam a medicação quando comparados aos que não utilizaram a droga testada. Não houve diferença estatisticamente significativa nas médias desta variável no final do estudo.

Com relação às coletas de sangue, nos grupos 1, 2 e 3 foram retirados, em média $21,9 \mathrm{ml}, 22,9 \mathrm{ml}$ e $25,1 \mathrm{ml}$ de sangue, respectivamente, durante todo o seguimento do trabalho. Não houve diferença estatisticamente significativa entre os três grupos $(\mathrm{p}=0,839)$.

O volume de sangue transfundido no grupo 1 foi de 4,6 $\mathrm{ml}$ para cada paciente; no grupo 2, de 9,6 $\mathrm{ml}$ e no grupo 3 , de 17,6 ml. Estes achados, apesar de indicarem uma tendência de o grupo 3 (controle) receber um volume de sangue transfundido maior por paciente, não foram estatisticamente significativos $(\mathrm{p}=0,156)$.

No que diz respeito ao número de TSs por paciente, a média dessa variável no grupo 1 foi de 0,33 , no grupo 2 , de 0,64 e no grupo 3 de $1,62(p=0,091)$. Parece haver diferença entre os grupos, mas não foi atingido o nível de significância estatística estabelecido (alfa 5\%).

No tocante à variável transfusão excessiva, conceituada aqui como duas ou mais transfusões por paciente, houve uma diferença significativa entre os grupos $(\mathrm{p}=0,043)$. Foi considerada transfusão excessiva mais de uma TS por

Tabela 2 - Médias e desvios padrões de peso, comprimento e perímetro cefálico no início e no final do estudo e médias de aumento destas varáveis

\begin{tabular}{lccccccc}
\hline Características & \multicolumn{2}{c}{$\begin{array}{c}\text { Grupo 1 } \\
\mathbf{n}=\mathbf{1 5}\end{array}$} & \multicolumn{2}{c}{$\begin{array}{c}\text { Grupo 2 } \\
\mathbf{n}=\mathbf{1 4}\end{array}$} & \multicolumn{2}{c}{$\begin{array}{c}\text { Grupo 3 } \\
\mathbf{n}=\mathbf{1 3}\end{array}$} & \multirow{2}{*}{ P* } \\
& Média & DP & Média & DP & Média & DP & \\
\hline Peso final (g) & 2012,7 & 45 & 1980,7 & 73,8 & 2005,4 & 43,5 & 0,290 \\
Ganho Ponderal (g/dia) & 24,7 & & 24,3 & 4,2 & 26,2 & 6 & 0,652 \\
Comprimento final (cm) & 42,8 & 1,3 & 42,5 & 1,2 & 42,2 & 1,01 & 0,409 \\
Aumento do comprimento (cm) & 6,3 & 3,8 & 5 & 2,4 & 4,6 & 3 & 0,347 \\
Perímetro cefálico final (cm) & 31,5 & 0,9 & 31,8 & 1,08 & 31,6 & 0,64 & 0,585 \\
Aumento do perímetro cefálico (cm) & 4,6 & 1,8 & 4,5 & 2,2 & 4,6 & 2,2 & 0,981 \\
\hline
\end{tabular}


Tabela 3 - Médias e desvios padrões dos valores hematimétricos no início do estudo: hematócrito, hemoglobina, leucócitos totais, reticulócitos, plaquetas e ferritina

\begin{tabular}{lccccccc}
\hline Características & \multicolumn{2}{c}{$\begin{array}{c}\text { Grupo 1 } \\
\mathbf{n}=\mathbf{1 5}\end{array}$} & \multicolumn{2}{c}{$\begin{array}{c}\text { Grupo 2 } \\
\mathbf{n}\end{array}$} & $\mathbf{1 4}$ & \multicolumn{2}{c}{ Grupo 3 } \\
$\mathbf{n}=\mathbf{1 3}$ & \multirow{2}{*}{$\mathbf{P}^{*}$} \\
& Média & DP & Média & DP & Média & DP & \\
\hline Hematócrito (\%) & 36,6 & 4,5 & 36,5 & 3,7 & 36,5 & 5 & 0.998 \\
Hemoglobina (g/dl) & 12 & 1,5 & 12 & 1,2 & 12 & 1,9 & 0,981 \\
Leucócitos totais & 12.120 & 4.346 & 10.550 & $4.672,5$ & 12.720 & $5.807,2$ & 0,498 \\
Reticulócitos (\%) & 2,45 & 1,4 & 2,12 & 0,9 & 3,02 & 2,5 & 0,419 \\
Plaquetas & 381.266 & 200.033 & 392.643 & 120.975 & 490.770 & 170.106 & 0,105 \\
Ferritina (ng/ml) & 422,5 & 284 & 269 & 231,4 & 501,5 & 428 & 0.170 \\
& & & & & & & \\
\hline
\end{tabular}

paciente, com base na exposição a múltiplos doadores, que os autores consideraram mais de um por RN. No grupo 1, dos 15 pacientes que receberam, diariamente, a medicação testada, em apenas um $(6,7 \%)$ foi necessário fazer transfusão excessiva de sangue. Já no grupo 2 , três $(21,4 \%)$ pacientes dos 14 que utilizaram a droga em estudo duas vezes por semana foram transfundidos excessivamente, enquanto no grupo 3 , para o qual a EPO não foi administrada, dos 13 recém-nascidos, cinco $(38,5 \%)$ foram transfundidos em excesso. Apesar de as crianças estudadas no grupo 1 apresentarem uma proporção bem menor de transfusões excessivas $(6,7 \%)$ do que as crianças do grupo $2(21,4 \%)$, não foi detectada diferença significativa entre os dois grupos $(\mathrm{p}=0,33)$.

\section{Discussão}

A partir de um conhecimento mais profundo da hematopoese na vida fetal e neonatal e dos fatores que a regulam, em especial a EPO, chegou-se a uma nova conceituação e abordagem das anemias, entre elas, a anemia da prematuri- dade. A EPO, uma citoquina cuja função é estimular a eritropoese, está disponível para uso e estudos clínicos desde 1985, e era esperado que, com seu uso, a necessidade de TSs seria eliminada ${ }^{19}$.

No nosso estudo houve uma preocupação inicial em avaliar as características gerais da população. A constatação de que essas se distribuíram de forma semelhante entre os três grupos, conforme observado na Tabela 1 , minimiza os eventuais vícios de seleção que poderiam interferir nos resultados encontrados.

Em relação à adequação do peso à idade gestacional, os três grupos foram semelhantes. A grande maioria dos PMTs que participaram do estudo foram considerados adequados para a idade gestacional. Este dado é bastante importante, pois segundo Brown e colaboradores ${ }^{16}$, a idade gestacional, principalmente quando abaixo de 30 semanas, é o melhor valor de predição de necessidade de TS após as duas semanas de vida. Portanto, um RN pequeno para a idade gestacional (peso desproporcionalmente baixo para a idade gestacional), teoricamente, teria um menor risco de necessitar TS.

Tabela 4 - Médias e desvios padrões dos valores hematimétricos: hematócrito, hemoglobina, ferritina e reticulócitos ao término do estudo e de reticulócitos na segunda semana de tratamento

\begin{tabular}{|c|c|c|c|c|c|c|c|}
\hline \multirow[t]{2}{*}{ Características } & \multicolumn{2}{|c|}{$\begin{array}{c}\text { Grupo } 1 \\
\mathrm{n}=15\end{array}$} & \multicolumn{2}{|c|}{$\begin{array}{c}\text { Grupo } 2 \\
n=14\end{array}$} & \multicolumn{2}{|c|}{$\begin{array}{c}\text { Grupo } 3 \\
\mathbf{n}=13\end{array}$} & \multirow[t]{2}{*}{$\mathbf{P}^{*}$} \\
\hline & Média & DP & Média & DP & Média & DP & \\
\hline Hematócrito (\%) & 29,7 & 3,6 & 28,7 & 2,9 & 25,1 & 3,3 & 0,002 \\
\hline Hemoglobina (g/dl) & 9,5 & 1 & 9,3 & 0,9 & 8,3 & 1,1 & 0,0011 \\
\hline Ferritina (ng/ml) & 146,5 & 132 & 93,5 & 63,8 & 210,2 & 284,1 & 0,254 \\
\hline $\begin{array}{l}\text { Reticulócitos ao término } \\
\text { do estudo (\%) }\end{array}$ & 10 & 5,4 & 9,3 & 3,2 & 6,8 & 5,1 & 0,160 \\
\hline $\begin{array}{l}\text { Reticulócitos na segunda } \\
\text { semana de tratamento }(\%)\end{array}$ & 11 & 5,9 & 10,9 & 4,4 & 4,1 & 2 & 0,0001 \\
\hline
\end{tabular}


Observou-se uma estabilidade clínica, cujos critérios foram descritos em material e métodos, de todos pacientes, assim como está nas publicações da maioria dos autores $^{1-3,9,22,27-29}$. Apesar de alguns estudos não terem evidenciado correlação do Ht com a manifestação respiratória $^{4}$, um dos critérios estabelecidos por Strauss e colaboradores $^{30}$, em 1990, para indicar a TS seria a manutenção do $\mathrm{Ht}$ em $40 \%$ naqueles pacientes com doenças respiratórias. Portanto, teoricamente, caso houvesse uma diversidade clínica e ventilatória muito grande entre os grupos, como por exemplo, a presença de RNs com maior necessidade de suporte ventilatório nos grupos $1 \mathrm{e} 2$, que receberam a EPO, poderia se concluir erroneamente que a medicação testada não diminui a quantidade de TSs, pois os pacientes desses grupos teriam sido mais transfundidos, provavelmente pela maior gravidade clínica.

O desenvolvimento pôndero-estatural dos RNs ao longo do estudo foi semelhante nos três grupos, concordando com os resultados descritos na literatura ${ }^{3,24}$. Não houve diferenças estatisticamente significativas em relação às médias de peso, comprimento e perímetro cefálico no final do trabalho, assim como descrito por Bechensteen e colaboradores em $1993^{1} \mathrm{e} \mathrm{em} 1996^{31}$. Em nenhum momento do estudo ocorreram diferenças quanto ao ganho de peso, comprimento e perímetro cefálico médio entre os grupos que receberam a EPO e o grupo que não fez uso da medicação. A razão dessa observação não é clara. Sabe-se que a estimulação do aumento da eritropoese implica aumento da demanda de proteínas e vitaminas, não adequadamente suprida pela nutrição desses RNs. Apesar de Shannon e colaboradores ${ }^{29}$, em 1990, terem demonstrado um ganho ponderal mais rápido nos pacientes que foram tratados com EPO, na maioria dos ensaios clínicos 1,3,10,24,27 não foi detectada diferença significativa no ganho de peso entre os grupos tratados ou controle. $\mathrm{O}$ aumento de peso diário nos grupos 1, 2 e 3 do nosso estudo foi respectivamente, de 24,7g, 24,3g e 26,2g, muito semelhante ao estudo de Meyer e colaboradores no qual o ganho ponderal variou de $23,2 \mathrm{~g}$ (nos pacientes tratados com EPO) e 22,7g (nos RNs que não receberam a medicação), enquanto Bechensteen e colaboradores obtiveram um aumento, em média, de 18,5g/dia. Em nossa pesquisa, todos os PMTs foram alimentados com leite artificial, próprio para PMTs, e receberam em média $121,8 \mathrm{cal} / \mathrm{kg} / \mathrm{dia}$. Halperin e colaboradores ${ }^{7}$ também não encontraram diferenças estatisticamente significativas quanto à variável em questão.

Ao avaliarmos os valores hematológicos iniciais $-\mathrm{Ht}$, $\mathrm{Hb}$, contagem de reticulócitos, leucócitos totais, plaquetas e ferritina dos RNs -, conforme demostrado na Tabela 3, não encontramos diferenças estatisticamente significativas entre os grupos. Dessa forma, as diferenças observadas nas contagens finais de $\mathrm{Ht}$ e $\mathrm{Hb}$ não podem ser explicadas pelas contagens iniciais dessas variáveis. Os resultados mostraram que os $\mathrm{Hts}$ finais foram significativamente maiores nos pacientes que receberam EPO, diariamente ou fracionada em duas vezes na semana, quando comparados aos do grupo controle, concordando com vários estudos da literatu-

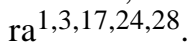

Quanto à contagem absoluta de reticulócitos, os resultados mostraram claramente que, ao final da segunda semana do estudo, esta foi significativamente maior nos pacientes que receberam a medicação quando comparada à dos que não a utilizaram, o que evidencia claramente que os PMTs são capazes de responder à EPO exógena com um aumento da eritropoese. Achado semelhante foi relatado por Bechesteen e colaboradores ${ }^{1}$. Em relação ao pico da contagem de reticulócitos, este foi encontrado ao término da segunda semana de tratamento, sendo a diferença estatisticamente significativa entre a contagem inicial de reticulócitos e ao final da segunda semana de administração da medicação. Esses achados foram demonstrados pelos autores do presente estudo e por outros pesquisadores ${ }^{16,22}$. Quando avaliamos essa variável ao término do trabalho, apesar de haver uma tendência de a contagem dos reticulócitos ser menor no grupo 3, não houve diferença estatisticamente significativa entre os três grupos. Uma das possíveis justificativas para esse achado é o fato de poder ter ocorrido uma parada de produção da EPO endógena, decorrente, dentre outros fatores, do aumento da $\mathrm{Hb}$, ou mais precisamente, da ausência de diminuição da mesma necessária para estimular a EPO.

No tocante à contagem total de leucócitos e plaquetas, não foi detectada diferença significativa entre os grupos, em nenhuma das semanas estudadas, assim como já referido por alguns autores $1,3,9,10,27,32$.

No Brasil, assim como nos Estados Unidos, não há unaminidade quanto ao uso da EPO para o tratamento da anemia da prematuridade. Segundo Doyle e colaboradores $^{33}$, os ensaios clínicos com EPO objetivavam estudar PMTs, geralmente com idade gestacional de até 34 semanas e com até $1500 \mathrm{~g}$ de peso ao nascer, mas constata-se uma variedade na idade de início do tratamento. A EPO foi administrada mais precocemente, a partir da primeira semana de vida, em alguns estudos ${ }^{10,17,23}$ e mais tardiamente, em média com 21 dias de vida, em outros trabalhos $1,3,18,24,28,29$. Neste estudo a média de idade de início do tratamento foi de aproximadamente 15 dias. O início do tratamento por volta da $2^{\mathrm{a}}$ a $3^{\mathrm{a}}$ semanas de vida e a sua continuação durante 6 a 8 semanas parece ser o esquema de administração que tem se mostrado efetivo segundo as revisões bibliográficas. Por outro lado, ainda não está claramente estabelecida a utilidade da administração de EPO desde as primeiras horas de vida a fim de diminuir a necessidade de TSs nos primeiros 15 dias de vida.

O esquema terapêutico ideal para uso de EPO permanece indeterminado, devido às diferentes doses semanais e frequiência de uso utilizadas nesses ensaios ${ }^{6}$. As doses variam de $150-1400 \mathrm{U} / \mathrm{kg} / \mathrm{semana}$, e a freqüência de utilização, de 2-7 dias por semana. Os autores concordam que o tratamento com EPO em doses maiores ou iguais a 500U/ $\mathrm{kg} /$ semana não parece trazer complicações, induz uma marcada estimulação da eritropoese e diminui considera- 
velmente a necessidade de TSs nos RNs. Halperin e colaboradores ${ }^{9}$, em 1992, encontraram uma resposta dose-dependente, mas ainda há dúvidas quanto à existência de um efeito positivo na eritropoese com a administração mais freqüente de EPO. Apesar de Brown e colaboradores ${ }^{26}$, bem recentemente, terem demonstrado esse efeito, não foi encontrada redução significativa no número de TSs desses RNs, nem uma diferença entre os Hts do grupo que recebeu a EPO com menores intervalos de administração (5 vezes por semana) quando comparado ao grupo no qual as doses foram administradas com menor freqüência semanal (2 vezes por semana). No nosso estudo, com o propósito de comparar o uso diário da EPO e o uso da mesma dose semanal, mas dividida em duas vezes, foi feita uma análise estatística entre os grupos 1 e 2. Apesar de os PMTs do grupo 1 apresentarem uma proporção bem menor $(6,7 \%)$, de duas ou mais TSSs por paciente que os RNs do grupo 2 $(21,4 \%)$, não foi detectada diferença significativa entre estes dois grupos, provavelmente pelo pequeno tamanho da amostra estudada. Para podermos detectar uma diferença significativa entre os dois grupos de recém-nascidos prematuros quanto à diminuição de número de transfusões excessivas, haveria a necessidade de estudar, pelo menos, uma amostra quase duas vezes maior que a estudada -25 pacientes em cada grupo.

A eficácia da EPO em reduzir as TSs realizadas em PMTs foi demonstrada em vários ensaios clíni$\cos ^{1-3,10,17,22,23,26-29,32}$. Neste estudo, apesar de os dados indicarem que os pacientes sem o tratamento com EPO tendem a necessitar de um maior volume de sangue transfundido, o teste estatístico não evidenciou diferença significativa entre os grupos. Por outro lado, houve uma menor proporção de pacientes transfundidos excessivamente no grupo 2, tratado com EPO em duas doses por semana, do que no grupo 3 , sem a medicação $(21,4 \%$ versus $38,5 \%$ respectivamente) - diferença estatisticamente significativa. A EPO é útil, mas não deve ser utilizada como estratégia isolada para diminuir a exposição dos RNs às TSs. Surge claramente a necessidade de estabelecer critérios de transfusões onde há uma política de transfundir somente em situações muito necessárias com benefícios evidentes. Devese ainda lembrar de restringir as coletas de sangue para análises. Solicitar exames de forma racional e sempre que possível usar microtécnicas nas coletas de sangue.

Sabe-se que a eritropoese, em qualquer idade, é dependente de um aporte nutricional adequado e, particularmente, da ingestão de proteína e ferro, sendo a restrição desses nutrientes uma limitação significativa ${ }^{1,34}$. Essa dependência é pronunciada, principalmente, na fase em que se encontram esses pacientes, pois é nela que se verifica um crescimento mais rápido do que em qualquer outra etapa da $v \operatorname{vida}^{33}$. Com base neste conhecimento, em nossa pesquisa houve uma preocupação quanto ao aporte de leite administrado aos RNs. Como já foi apresentado, a análise dos aportes calórico e proteíco não evidenciou diferença estatisticamente significativa entre os três grupos. Talvez esti- vesse indicada uma ingestão protéica um pouco maior, $3 \mathrm{~g} /$ $\mathrm{kg} / \mathrm{dia}$, como foi utilizado por Bechensteen e colaboradores $^{1}$. Mesmo não tendo sido definida a quantidade de proteína adequada para estimular a eritropoese durante $o$ tratamento com EPO, é prudente ajustar a ingestão proteíca à quantidade adequada para o crescimento esperado do $\mathrm{RN}^{34}$. Quanto ao aporte de ferro, esses mesmos autores utilizaram $18 \mathrm{mg} / \mathrm{dia}$, aumentando para $36 \mathrm{mg} /$ dia caso houvesse diminuição da concentração de ferro ${ }^{34}$. Eles justificam que o aumento da eritropoese ocorra devido ao alto aporte de proteína, pois a dose da EPO utilizada foi relativamente baixa, 300U/kg/semana. Em nosso trabalho, apesar de não terem sido utilizadas suplementações de ferro e proteína tão altas quanto as adotadas por Bechenteen e colaboradores $^{35}$, a dose de EPO empregada por nós, de $700 \mathrm{U} / \mathrm{kg} / \mathrm{semana}$, foi mais que o dobro da administrada por eles e tanto o aumento da contagem dos reticulócitos no final da segunda semana de tratamento, como os valores de $\mathrm{Ht}$ e $\mathrm{Hb}$ ao término do estudo foram maiores nos grupos tratados com EPO quando comparados aos controles.

Os PMTs apresentam baixos estoques de ferro, pela diminuição do tempo de gestação e baixa ingestão durante a prolongada internação hospitalar a que são submetidos. Foi demonstrado que o uso de EPO aumenta essa deficiência, de forma que a suplementação de ferro se faz necessária dentro do esquema de tratamento com EPO. O não uso de ferro foi relacionado a falhas terapêuticas, doses baixas de suplementação levaram a depleção de estoques orgânicos de ferro até à anemia rebote ${ }^{22}$. Atualmente estamos em condições de afirmar que a quantidade de ferro a ser administrada para que o tratamento com doses elevadas de EPO seja efetivo não deve ser inferior a $6 \mathrm{mg} / \mathrm{kg} / \mathrm{dia}^{18,27}$. Há uma grande variedade na dose de ferro nos ensaios clínicos, dificultando essa interpretação. Como sugerido, existe a probalidade de que a quantidade de ferro disponível seja o principal fator limitante das doses de EPO a serem administradas ${ }^{18}$. Os aspectos relativos à via de administração e ao momento de iniciar a suplementação do ferro permanecem ainda em discussão. A grande maioria dos artigos já descritos neste trabalho utiliza a via oral e a dose de 2 a $8 \mathrm{mg} / \mathrm{kg} /$ dia do elemento referido. Ohls e colaboradores $^{32}$ utilizaram a via intravenosa para a suplementação de ferro, em associação com a nutrição parenteral total. Um estudo realizado por Meyer e colaboradores ${ }^{36}$ demonstrou que altas doses de ferro administradas por via oral ou parenteral foram suficientes para manter a eritropoese estável nos pacientes, apesar do estoque de ferro do grupo que o utilizou por via oral ter sido mais depletado. A partir dessas informações, os autores deste estudo sugerem a utilização da via intravenosa para administração do ferro somente naqueles RNs que apresentarem níveis muito baixos de ferritina.

Após avaliação dos resultados dos estudos de Brown e colaboradores ${ }^{26,34}$, consideramos os níveis de ferritina mais confiáveis que o ferro sérico ou a saturação de transferrina para esta interpretação. Os maiores níveis de ferri- 
tina obtidos no nosso estudo foram observados no grupo controle (sem diferença estatisticamente significativa) e ocorreram provavelmente devido à mobilização do ferro pelos RNs do grupo tratado com EPO. Como apresentado anteriormente nos resultados, houve um declínio, já esperado, desta variável ao longo do estudo, mas o mesmo não foi diferente estatisticamente entre os três grupos, concordando com o constatado por alguns autores $23,27,34,35$. Esse achado talvez possa ser explicado pela adequada suplementação de ferro, utilizada neste estudo, o que não permitiu que houvesse uma queda tão pronunciada do mesmo no decorrer do tratamento com EPO.

A via padrão de administração de EPO é a subcutânea ${ }^{32}$, sendo esta a utilizada em nosso estudo e na grande maioria dos ensaios clínicos realizados em RNs com anemia da prematuridade ${ }^{1,9,16,17,22-24,27-29,31,34,35}$. A via intravenosa foi utilizada apenas por poucos autores ${ }^{32,37}$.

Outro aspecto importante a considerar são os possíveis efeitos colaterais da droga, como plaquetocitose e granulocitopenia. Nenhum efeito tóxico decorrente do uso dessa medicação foi demonstrado em nosso trabalho, concordando com os achados de outros autores ${ }^{1,22,27,28,32}$. Quanto aos possíveis efeitos a longo prazo do uso de EPO, não se obteve dados para adequadas conclusões e, na literatura, não há, até o momento, nenhum estudo prospectivo com esta abordagem.

Concluímos que a administração de EPO, na dose semanal de 700U/kg, nos PMTs com peso de nascimento até $1.550 \mathrm{~g}$ e idade gestacional de até 33 semanas estimula a eritropese e reduz de forma significativa o número de TSs excessivas, diminuindo a exposição desses RNs aos potenciais riscos transfusionais. A partir dos resultados obtidos com o nosso estudo e com a revisão de literatura realizada indicamos, rotineiramente, nos PMTs com idade gestacional até 33 semanas, peso de nascimento até $1.550 \mathrm{~g}$, estáveis clinicamente, o uso de EPO, iniciando ao redor do $15^{\circ}$ dia de vida, na dose de 700U/kg/semana, via subcutânea. Para avaliarmos a posologia ideal há a necessidade de um estudo com maior número de pacientes. Ressaltamos que a utilização dessa medicação, nessa dose indicada, mostrou-se segura e bem tolerada.

\section{Referências bibliográficas}

1. Bechensteen AG, Hågå P, Halvorsen $\mathrm{S}$, Whiterlaw A, Liestøl K, Lindermann R, et al. Erythropoietin, protein, and iron supplementation and the prevention of anaemia of prematurity. Arch Dis Child 1993;69:19-23.

2. Beck D, Masserey E, Meyer M, Calame A. Weekly intravenous administration of recombinant human erythropoietin in infants with the anaemia of prematurity. Eur J Pediatr 1991;150:767-72.
3. Shannon KM, Mentzer WC, Abels RI, Freeman P, Newton N, Thompson D, et al. Recombinant human erythropoietin in the anemia of prematurity: results of a placebo-controlled pilot study. J Pediatr 1991;118:949-55.

4. Keyes WG, Donohue PK, Spivak JL, Jones MD, Oski FA. Assessing the need for transfusion of premature infants and role of hematocrit, clinical signs, and erythropoietin level. Pediatrics 1989;84:412-7.

5. Stockman III JA, Garcia JF, Oski FA. The anemia of prematurity factors governing the erythropoietin response. N Engl J Med. 1977;296:647-50.

6. Strauss RG. Neonatal anemia: pathophysiology and treatment. Immunological Investigations 1995;24:341-51.

7. Halpérin DS. Use of recombinant erythropoietin in treatment of the anemia of prematurity. Am J Pediatr Hematol Oncol 1991;13:351-63.

8. Attias D. Pathophysiology and treatment of the anemia of prematurity. Journal of Pediatric Hematology/Oncology 1995;17:13-8.

9. Halpérin DS, Félix M, Wacker P, Lacourt G, Babel J-F, Wyss M. Recombinant human erythropoietin in the treatment of infants with anaemia of prematurity. Eur J Pediatr 1992;151:661-7.

10. Soubasi V, Kremenopoulos G, Diamandi E, Tsantali C, Tsakiris D. In which neonates does early recombinant human erythropoietin treatment prevent anemia of prematurity? Results of a randomized controlled study. Ped Res 1993;34:675-9.

11. Widness JA, Seward VJ, Kromer IJ, Burmeister LF, Bell EF, Strauss RG. Changing patterns of red blood cell transfusion in very low birth weight infants. J Pediatr 1996;129:680-7.

12. Brown MS, Garcia JF, Phibbs RH, Dallman PR. Decreased response of plasma immunoreactive erythropoietin to "avaiable oxygen" in anemia of prematurity. J Pediatr 1984;105:793-8.

13. Phibbs RH. 18th Annual International Neonatal Respiratory Care Symposium entitled "Oxygen and the Neonate", November, 1994. University of Miami School of Medicine, Miami, Florida.

14. Christensen RD. Recombinant erythropoietic growth factors as an alternative to erythrocyte transfusion for patients with "anemia of prematurity" [commentary]. Pediatrics 1989;83:793-6.

15. Strauss RG. Neonatal anemia: pathophysiology and treatment. In: Wilson SM, Levvit JS, Strauss RG, eds. Improving transfusion practice for pediatric patients. Arlington VA: American Association of Blood Banks; 1991. p.1-17.

16. Brown MK, Berman ER, Luckey D. Prediction of the need for transfusion during anemia of prematurity. J Pediatr 1990; 116:773-8.

17. Carnielli V, Montini G, Da Riol R, Dall'Amico R, Cantarutti F. Effect of high doses of human recombinant erythropoietin on the infants for blood transfusions in preterm infants. J Pediatr 1992; 121:98-102.

18. Donato H, Vain N, Rendo P. Tratamiento com eritropoyetina humana recombinante en período neonatal: indicaciones y controversias. Arch Argentinos de Pediatria 1996;94:28-45.

19. Juul SE. Erythropoietin in the neonate. Curr Probl Pediatr 1999; 29:133-49.

20. Rhondeau SM, Christensen RD, Ross MP, Rothstein G, Simmons MA. Responsiveness to recombinant human erythropoietin of marrow erythroid progenitors from infants with the "anemia of prematurity". J Pediatr 1988;112:935-40.

21. Shannon KM, Naylor GS, Torkildson JC, Clemons GK, Schaffner $\mathrm{V}$, Goldman SL, et al. Circulating erythroid progenitors in the anemia of prematurity. N Engl J Med. 1987;317:728-33.

22. Halpérin DS, Wacker P, Lacourt G, Félix M, Babel J-F, Aapro M, Wyss M. Effects of recombinant human erythropoietin in infants with the anemia of prematurity: a pilot study. J Pediatr 1990;116:779-86. 
23. Obladen M, Maier R, Segerer H, Grauel EL, Holland BM, Stewart G, et al. Efficacy and safety of recombinant human erythropoietin to prevent the anaemias of prematurity European randomized multicenter trial. In: Gurland HJ, Moran J, Samtieben W, Scigalla P, Wieczorek L, eds. Erythropoietin in renal and non-renal anemias update on basic research and clinical applications. Karger; 1991 p.314-26.

24. Shannon KM, Keith III JF, Mentzer WC, Ehrenkranz RA, Brown MS, Widness JA, et al. Recombinant human erythropoietin stimulates erythropoiesis and reduces erythrocyte transfusions in very low birth weight preterm infants. Pediatrics 1995;95:1-8.

25. Obladen M, Maier RF. Erythropoietin therapy in preterm infants. In: Hansen TN, McIntosh N, eds. Current topics in neonatology. Philadelphia: Saunders; 1997.p108-24.

26. Brown MS, Keith JF. Comparison between two and five doses a week of recombinant human erythropoietin for anemia of prematurity: a randomized trial. Pediatrics 1999;104:210-5.

27. Soubasi V, Kremenopoulos G, Diamanti E, Tsantali C, Sarafidis K, Tsakiris D. Follow-up of very low birth weight infants after erythropoietin treatment to prevent anemia of prematurity. J Pediatr 1995;127:291-7.

28. Bader D, Blondheim O, Jonas R, Admoni O, Abend-Winger M, Reich D, et al. Decrease ferritin levels, despite iron supplementation, during erythropoietin therapy in anaemia of prematurity. Acta Pædiatr Scand 1996;85:497-501.

29. Shannon KM, Mentzer WC, Abels RI, Wertz M, Tayer-Moriyama J, Li WY, et al. Enhancement of erythropoiesis by recombinant human erythropoietin in low weight infants: a pilot study. $\mathrm{J}$ Pediatr 1992;120:586-92.

30. Strauss RG, Sacher JF, Blazina VS, Blanchette VS, Schloz LM, Butch SH, et al. Commentary on small-volume red cell transfusions for neonatal patients. Transfusion 1990;30:565-70.

31. Bechensteen AG, Halvorsen S, Hågå P, Cotes PM, Liestøl K. Erythropoietin (Epo), protein and iron supplementation and the prevention of anaemia of prematurity: effects on serum immunoreactive Epo, growth and protein and iron metabolism. Acta Pædiatrics 1996;85:490-5.
32. Ohls RK, Veerman MW, Christensen RD. Pharmacokinetics and effectiveness of recombinant erythropoietin administered to preterm infants by continuous infusion in total parenteral nutrition solution. J Pediatr 1996;128:518-23.

33. Doyle JJ. The role of erythropoietin in the anemia of prematurity. Semin in Perinatol 1997;21:20-7.

34. Brown MS, Howard S. Effect of protein intake on erythropoiesis during erythropoietin treatment of anemia of prematurity. $\mathbf{J}$ Pediatr 1996;128:512-7.

35. Bechensteen AG, Hågå P, Halvorsen S, Liestøl K, Lindemann R, Whitelaw A. Effect of low and moderate doses of recombinant human erythropoietin on the haematological response in premature infants on a high protein and iron intake. Eur J Pediat 1997;156:56-61.

36. Meyer MP, Haworth C, Meyer JH, Commerford A. A comparison of oral and intravenous iron supplementation in preterm infants receiving recombinant erythropoietin. J Pediatr 1996; 129:258-63.

37. Miller DR. Anemias: general considerations. In: Miller DR. ed. Blood diseases of infancy and childhood. $7^{\text {th }}$ ed. Mosby- Year Book; 1995. p.111-39.

Endereço para correspondência:

Dra. Vera Lúcia L. Rocha

Av. Nilópolis, 473 - apto. 404

Porto Alegre - RS - CEP 90460-050

Fone: (51) 332.2204 / 9967.8002 